\title{
IMPORTÂNCIA DO DIÂMETRO DAS GOTAS DE PULVERIZAÇÃO NA DERIVA DE DEFENSIVOS AGRICOLAS EM APLICAÇÃO AÉREA
}

\author{
L.S.P. Cruz*; A. McCracken** e Y. Ozeki*** \\ * Pesq. Científico, Instituto Biológico, Caixa, \\ Postal 70 - 13.100 Campinas, SP. \\ ** Eng. ${ }^{\circ}$ Agr. ${ }^{\circ}$, FMC do Brasil S.A., S. Paulo, SP. \\ *** Eng. ${ }^{\circ}$ Agr. ${ }^{\circ}$, Serraria S.A. de Aviação \\ Agrícola, São Paulo, SP. \\ Recebido para publicação em 23.4.1979.
}

\section{RESUMO}

Foi conduzido um experimento no município paulista de Barra Bonita, com aplicações aéreas, onde foram comparadas as distâncias de deriva apresentadas por bicos de diversos tipos posicionados em ângulos diferentes. As aplicações foram feitas com uma solução de água contendo $0,1 \%$ de corante Lissamine Turquesa, com gas to de $301 /$ ha.

Os resultados mostraram a influência do ângulo formado pelos bicos em sua colocação na barra de pulverização e a influência do diâmetro das gotas de pulverização no efeito da deriva.

Os bicos cônicos D8-45 montados com capa - $\bullet$ Raindrop - reduziram significativamente o tamanho das gotas, o mesmo acontecendo com o bico $<\bullet$ Solid Stream, posicionado a $45^{\circ}$.

UNITERMOS: Gotas de pulverização, aplicação aérea, deriva.

\section{SUM MARY}

This paper deals with the droplet sizes and spray distribuition with several types of spray nozzles and different nozzles angles. The results were obtained by flight tests using a coloured dye.

It was clearly demonstrated that nozzle an gle has a very great effect on droplet size and hence spray drift.

Among the nozzles tested the D8-45 with the Raindrop cap offered the greatest advantages in reducing the number of small droplet and hence the risk of drift. Good results presented by the solid stream nozzle at $45^{\circ}$ angle, too.

KEYWORDS: droplet size, aerial application, drift.

\section{INT RODUÇÃO}

Um esforço razoável foi levado a efeito no passado para a comparação de deriva e objetivos a serem alcançados entre aplicações aéreas e terrestres (3). Os resultados alcançados por Ware et al. (6) mostraram que havia maiores possibilidades de se conseguir uma pulverização no alvo, portanto com pouca deriva, através de aplicações com equipamento terrestre e durante as primeiras horas da manhã. Porém, muitas vêzes essa aplicação terrestre torna-se impraticável, como é o caso de locais com clima muito úmido que não permite a utilização de equipamento terrestre, ou insetos e doenças que requerem um can-trole imediato, ou ainda, a falta de mão de obra treinada. Esses fatores existem nas condições da agricultura brasileira, como em culturas de soja. Convém salientar que $90 \%$ dos ins eticidas empregados na agricultura no estado de Arizona, EUA, são aplicados com avião (2). No Brasil, vem sendo muito empregada a pulverização aérea de herbicidas, principalmente em cana-de-açúcar; de arbusticidas, em pastagens; e, de desse-cantes, em soja.

Já foi bem estudada a teoria e mecânica do tamanho das gotas (8). 
Neste trabalho foi medida e comparada a variação de tamanho das gotas e portanto, a tendência de deriva dos diversos tipos de bicos posicionados em diferentes ângulos.

\section{MAT ERIAIS E MÉTODOS}

Com faixas de deposição de $10 \mathrm{~m}$ de largura, com passagem do apare- lho em seu sentido vertical, foi conduzido um ensaio em 11 de junho de 1976, no município de Barra Bonita, SP.

Os tratamentos constaram da pulverização de uma solução de água com $\mathbf{0 , 1 \%}$ de corante Lissamine Turquesa, com gasto de $301 /$ ha, com diferentes bicos de pulverização, posicionados em ângulos também diferentes, e sob as seguintes condições:

\begin{tabular}{|c|c|c|c|c|c|c|c|}
\hline Tratamento & Tipo de bico & $\begin{array}{c}\text { Ângulo } \\
\left({ }^{0}\right)\end{array}$ & $\begin{array}{l}\text { N. }{ }^{0} \text { de bicos na } \\
\text { barra de pulveri- } \\
\text { zaçâo }\end{array}$ & $\begin{array}{l}\text { Pressão } \\
\left(\mathbf{l b} / \text { pol }^{2}\right)\end{array}$ & $\begin{array}{l}\text { Velocidade } \\
\text { do vento } \\
(\mathbf{k m} / \mathbf{h})\end{array}$ & $\begin{array}{l}\text { Tempera- } \\
\text { tura }\left({ }^{\circ} \mathrm{C}\right)\end{array}$ & $\begin{array}{l}\text { Umidade } \\
\text { relativa } \\
\text { do ar }\end{array}$ \\
\hline 1 & Solid stream & 45 & 38 & 28 & 4 & 14 & 100 \\
\hline 2 & Solid stream & 45 & 38 & 28 & 5 a 9 & 17 & 85 \\
\hline 3 & Solid stream & 90 & 38 & 28 & 5 a 9 & 17 & 85 \\
\hline 4 & D8-45 Raindrop & 45 & 33 & 25 & 3 a 4 & 14 & 100 \\
\hline 5 & D8-45 Raindrop & 90 & 33 & 25 & 3 a 4 & 14 & 100 \\
\hline 6 & D8-45 Raindrop & 90 & 33 & 25 & 3 a 4 & 16 & 90 \\
\hline 7 & D8-45 Raindrop & 90 & 33 & 25 & 5 a 8 & 16 & 90 \\
\hline 8 & D8 -45 & 45 & 40 & 22 & 5 a 9 & 16 & 90 \\
\hline 9 & SS 80.15 & 90 & 21 & 20 & 4 a 6 & 17 & 85 \\
\hline
\end{tabular}

As pulverizações foram feitas com avião do tipo Cessna Ag-wagon, modelo "C", munido de sistema hidráulico de aplicação de líquido, com barra de pulverização e bicos.

Durante todo o período de aplicação as condições climáticas permaneceram estáveis, sem inversão. Muita nebulosidade e muita umidade do ar. As velocidades do vento foram registradas a $2,5 \mathrm{~m}$ de altura. A direção do vento formava um ângulo de $90^{\circ}$ com a linha de vôo.

A amostragem de gotas foi obtida colocando-se, em linha formando ângulo de $90^{\circ} \mathrm{com}$ a direção do vôo, pequenos bastões de madeira com cartões de papel "kromekote" de 2,50 x 0,80 m, grampeados em sua parte superior.

Para a análise dos resultados foram tomadas duas áreas de $0,01 \mathrm{~m}^{2}$ onde se fez a contagem e a medição das gotas. O tamanho das gotas foi estimado utilizando-se um gráfico adequado para a relação mancha de gota/tamanho de gota.

\section{RESULTADOS E DISCUSS ÃO}

Os resultados de número de gotas, porcentagem de gotas reunidas por grupo de tamanho e porcentagem de volume de pulverização por grupo de tamanho encontram-se nos quadros 1 e 2 e figura 1, respectivamente.

No quadro 3 são apresentadas as características de tamanho de gotas de diversos bicos sob condições de laboratório. A porcentagem de volume da pulverização na categoria de deriva, i.e., abaixo de $100 \mu$ de diâmetro, é demonstrada para cada tipo de bico, segundo Delavan Manf. Co. (2).

Reunindo-se em classes de porcentagens de volume os dados da figura 1 , obtem-se o quadro 4 .

A relação de deriva entre a velocidade do vento e o tamanho da gota é apresentada em trabalho de Eickestedt (3). 
Quadro 2. Porcentagem de gotas por grupos de tamanho, em micra, em ensaio com aplicaçáo aérea de defensivos agricolas com o aviảo Cessna Agwagon PT-IAX, em Barra Bonita, SP, em 1976.

\begin{tabular}{|c|c|c|c|c|c|c|c|c|c|c|}
\hline \multirow[b]{2}{*}{ Tipos de bicos e ângulos } & \multicolumn{10}{|c|}{ Porcentagem de gotas por grupo de tamanho } \\
\hline & $>\mathbf{5 0}$ & $>100$ & $>160$ & $>210$ & $>260$ & $>300$ & $>354$ & $>390$ & $>425$ & $<425$ \\
\hline 1 Solid stream $\left(45^{\circ}\right)$ & 47,50 & 22,70 & 10,60 & 6,00 & 3,60 & 2,15 & 2,15 & 1,30 & 1,00 & 2,70 \\
\hline 2 Solid stream $\left(45^{\circ}\right)$ & 43,00 & 20,90 & 14,80 & 7,40 & 3,70 & 2,30 & 1,23 & 1,72 & 1,23 & 3,60 \\
\hline 3 Solid stream $\left(90^{\circ}\right)$ & 41,40 & 26,00 & 14,90 & 7,70 & 4,30 & 2,40 & 1,35 & 0,77 & 0,48 & 0,63 \\
\hline 4 D8 -45 Raindrop $\left(45^{\circ}\right)$ & 35,00 & 22,00 & 13,00 & 9,20 & 5,20 & 1,70 & 2,10 & 1,80 & 1,30 & 7,70 \\
\hline 5 D8 $-45^{-}$Raindrop $\left(90^{\circ}\right)$ & 35,30 & 20,60 & 15,20 & 6,90 & 6,90 & 2,36 & 3,72 & 3,10 & 2,23 & 3,47 \\
\hline 6 D8-45 Raindrop $\left(90^{\circ}\right)$ & 34,30 & 21,80 & 15,10 & 9,90 & 7,70 & 3,91 & 2,66 & 1,74 & 1,12 & 1,70 \\
\hline 7 D8 -45 Raindrop $\left(90^{\circ}\right)$ & 38,80 & 25,60 & 13,20 & 6,60 & 5,80 & 2,90 & 2,10 & 1,74 & 0,83 & 2,30 \\
\hline $8 \mathrm{D} 8-45\left(45^{\circ}\right)$ & 31,70 & 30,00 & 18,60 & 8,20 & 4,40 & 2,30 & 1,53 & 1,20 & 0,55 & 1,64 \\
\hline 9 SS $80.15\left(90^{\circ}\right)$ & 55,90 & 22,50 & 11,40 & 5,20 & 2,53 & 0,89 & 0,63 & 0,25 & 0,21 & 0,38 \\
\hline
\end{tabular}



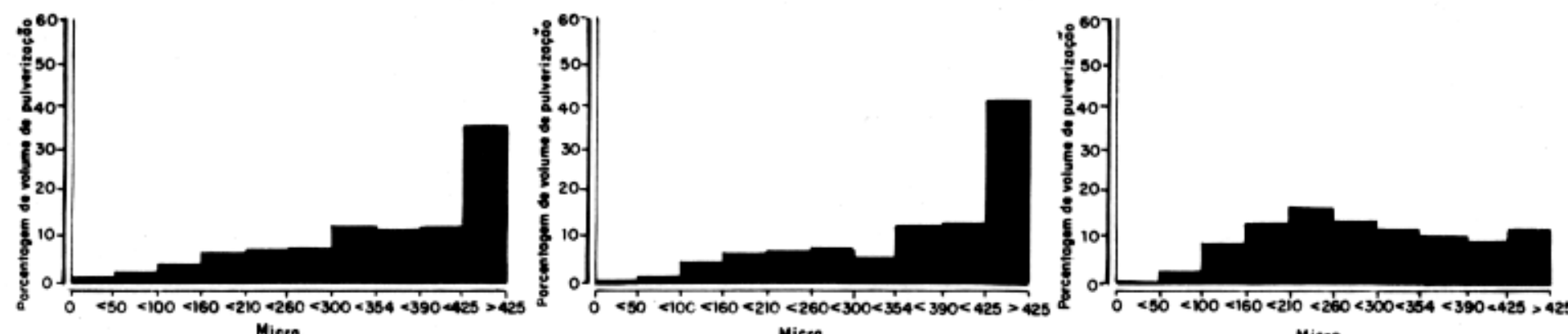

Tret.1- Bicos "Solid streom"(45")

Trat .2- Bicos "Solid stroem" (45")

Trat. 3-Bicos "Solid streom (90\%)
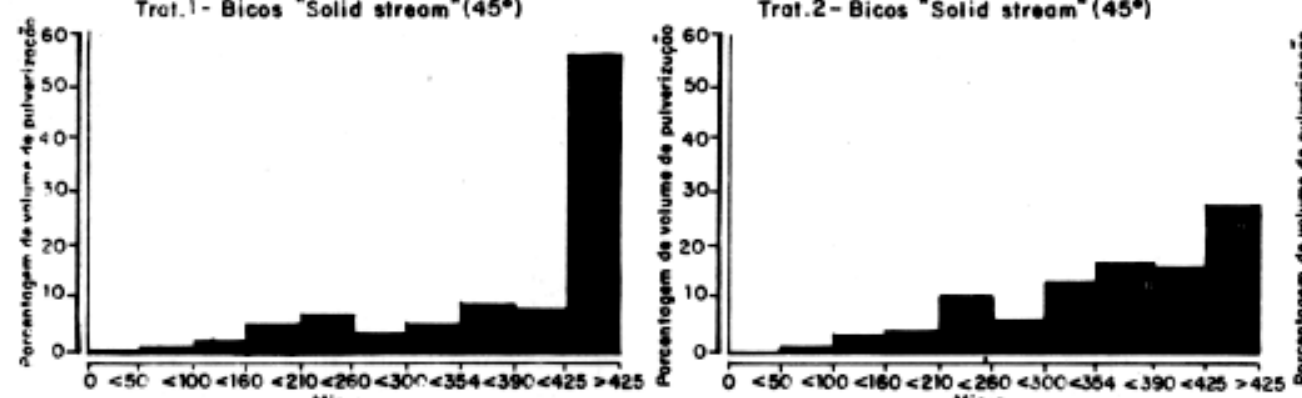

Trot. 5 - Biene DE. 4 E Faindr'p ! $30 \%$

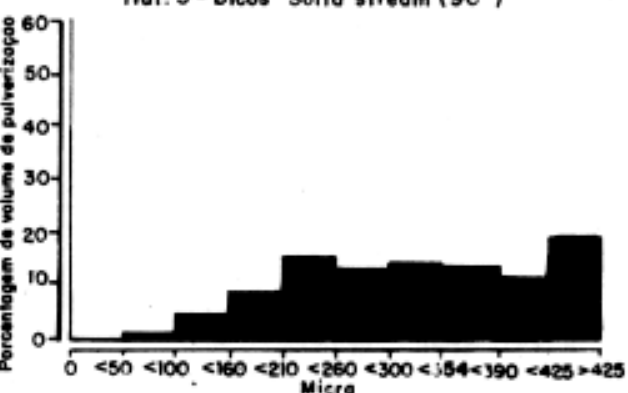

Trat. 6 - Bico" (A) 45 RcinArar (90\%)

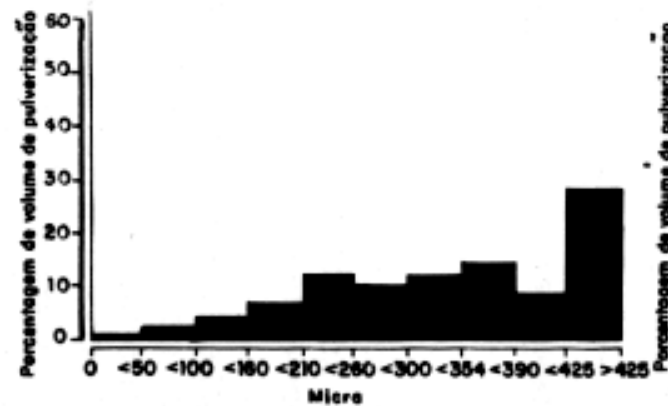

Trot. 7 - Bicos D8-45 Roindrop (95)

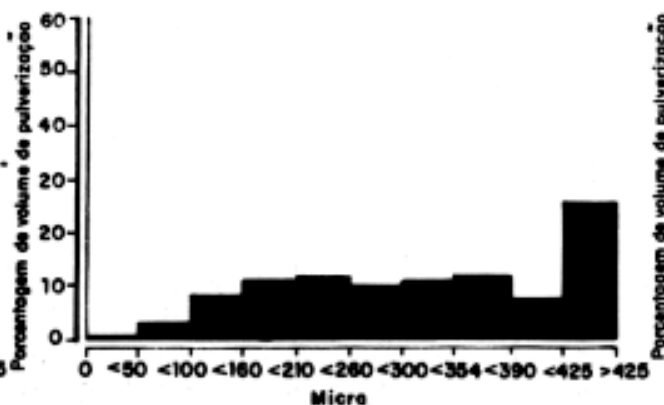

Tret. 8- Bicos D8-45 (45०)

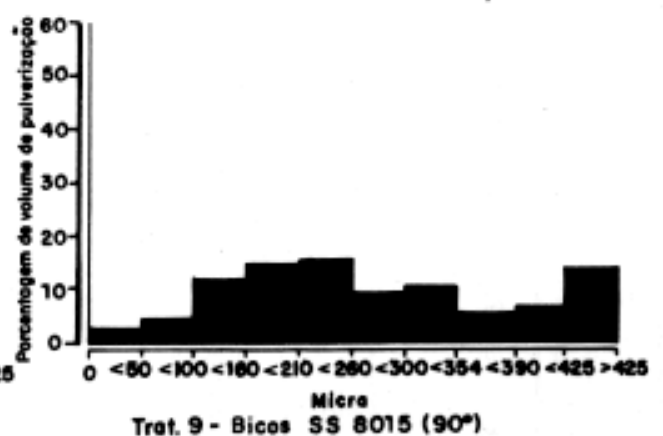

Trat. 9 - Bicos SS $8015\left(90^{\circ}\right)$

Figura 1. Porcentagem de volume de pulverizaçáo por grupo de tamanho em ensaio com aplicaçáo aérea de defensivos agrícolas com o avião Ces sna Agwagon PT-IAX, em Barra Bonita, SP, em 1976. 
Quadro 3. Comparação de tamanho de gotas entre quatro tipos de bicos com taxas de fluxo e pressão similares, em condições de laboratório.

\begin{tabular}{cccccc}
\hline $\begin{array}{c}\text { Padrão } \\
\text { de } \\
\text { pulverização }\end{array}$ & $\begin{array}{c}\text { Tipo e tamanho } \\
\text { de bico }\end{array}$ & Fluxo (G.P.M.) & Pressão (lb/pol $\left.{ }^{2}\right)$ & $\begin{array}{c}\text { Diâmetro médio } \\
(\mu \mathrm{m})\end{array}$ & $\begin{array}{c}\text { Volume de pul- } \\
\text { verização abaixo } \\
\text { de 100 } \mu(\%)\end{array}$ \\
\hline Flooding & D-1,5 & 0,30 & 40 & 210 & 13,0 \\
Leque & LF-3 & 0,30 & 40 & 202 & 15,5 \\
Cônico & DC4-25 & 0,29 & 40 & 195 & 15,9 \\
Cônico Raindrop & D4-25 & 0,29 & 40 & 410 & 0,8 \\
& $33974-2$ & & & & \\
& & & & &
\end{tabular}

Quadro 4. Porcentagem do volume de gotas de ensaio com aplicação aérea de defensivos agrícolas com o avião Cessna Agwagon, em Barra Bonita, SP, em 1976.

\begin{tabular}{|c|c|c|c|c|}
\hline $\begin{array}{c}\text { Tipos de bicos de pulverização } \\
\text { e ângulo }\end{array}$ & $\begin{array}{c}\text { Volume de pul- } \\
\text { verização abaixo } \\
\text { de } 100 \mu \\
(\%)\end{array}$ & $\begin{array}{l}\text { Razão de ten- } \\
\text { dência de de- } \\
\quad \text { riva }\end{array}$ & $\begin{array}{c}\text { Volume de pul- } \\
\text { verização de } \\
100 \text { a } 425 \mu \\
\text { (\%) }\end{array}$ & $\begin{array}{l}\text { Volume de pul- } \\
\text { verização acima } \\
\text { de } 425 \mu \\
(\%)\end{array}$ \\
\hline 1 Solid stream $\left(45^{\circ}\right)$ & 2,61 & 2,02 & 62,2 & 35,3 \\
\hline 2 Solid stream $\left(45^{\circ}\right)$ & 2,03 & 1,57 & 57,5 & 40,5 \\
\hline 3 Solid stream $\left(90^{\circ}\right)$ & 4,39 & 3,40 & 84,1 & 11,4 \\
\hline 4 D8-45 Raindrop $\left(45^{\circ}\right)$ & 1,29 & 1,00 & 42,4 & 56,4 \\
\hline 5 D8-45 Raindrop $\left(90^{\circ}\right)^{(1)}$ & 1,69 & 1,31 & 74,3 & 24,1 \\
\hline $8 \mathrm{D} 8-45\left(45^{\circ}\right)$ & 3,41 & 2,64 & 70,8 & 25,7 \\
\hline 9 SS $80.15\left(90^{\circ}\right)$ & 8,04 & 6,23 & 77,5 & 14,3 \\
\hline
\end{tabular}

(1) - Resultados médios dos tratamentos 5-6-7, com bicos D8-45 Raindrop a $\left(90^{\circ}\right)$

Segundo Seimour e Byrd (5), a duração da gota de pulverização depende da temperatura e da umidade relativa do ar.

Eickestedt (4) demonstra também que com qualquer aumento de temperatura ou redução de umidade relativa do ar, a deriva será mais acentuada.

Do exposto conclue-se que os dados apresentados na figura 1 demonstram que o bico "Raindrop" proporciona uma redução significativa na tendên- cia da deriva, assim como também o bico "Solid stream".

Este estudo confirmou o realizado por Ware et al. (7) onde ficou demons trado que o bico "Raindrop" depositou $25,7 \%$ a mais de pulverização no alvo quando comparado com os bicos tipo "Flooding".

Os resultados mostraram também, como pode ser visto na figura 1 , que 0 posicionamento dos bicos a $45^{\circ}$ oferece um aumento do tamanho das gotas 
quando comparado com o posicionamento a $90^{\circ}$.

Os bicos "Raindrop" e "Solid stream" oferecem vantagens especiais na aplicação de herbicidas, ou outros produtos químicos, onde a deriva possa causar danos à culturas vizinhas.

\section{LITERA TURA CITADA}

1. Akesson, N.B. e Yates, W.E. The use of aircaaft in agriculture. FAO. Boletim 94, Roma, 1974. 217p.

2. Delavan Manf. Co. Raindrop nozzles. Boletim 1493, West Des Moines, 1974. 10p.
3. Eickestedt, H.V. Die Applikations technik Von Insektiziden in Tropischen Feldkulturen. Höfchenbriefe, 1956. p.144-164.

4. Eickestedt, H.V. Agraflug in der DDR. Veb deutscher Landwirtschaftsverlaz. Berlin, 1974. 280p.

5. Seymoor, K.G. e Bird, B.C. Wind. Tunnel avaluation of spray drift potential. A.S.A.E. Trans., vol. 7, 1964.

6. Ware, G.W.; Aple, E.J.; Cahill, W.P.; Gerhart, P.D. e Frost, K.R. Pesticide drift. I - High Clearance V.3 aerial application of sprays. J. Econ. Entomol. 62:840-843, 1969.

7. Ware, G.W.; Cahill, W.P.; Gerhart, P.S. e Witt, J.W. Pesticide drift. IV - On target deposits from aerial application if insecticides. J. Econ. Entomol. 63: 1932-1933, 1970.

8. Yates, W.E. e Akesson, N.D. Reducing pesticide chemical drift. In: Valkenburg, W. Van, coord. Pesticide Formulations, New York, Marcel Dekker Inc., 1969, p.275-341. 